\title{
Das A und O ist die Allergie-Diagnostik
}

E ntsprechend dem Querschnittscharakter der Allergologie, die sich nicht an den Grenzen der einzelnen Organfächer orientiert, schrieben 41 ausgewiesene Fachleute aus der Pneumologie, HNO-Heilkunde, Dermatologie, Umweltmedizin und Pädiatrie sowie der klinischen Immunologie/Rheumatologie für dieses Werk.

Der erste Teil stellt die verschiedenen diagnostischen Methoden dar:

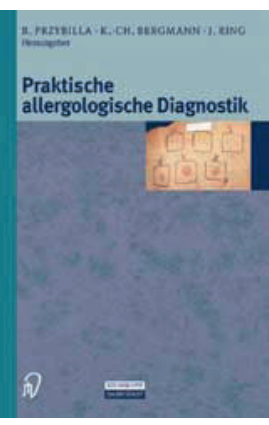

Przybilla, Bergmann, Ring, Hrsg. Praktische allergologische Diagnostik. Darmstadt: Steinkopf, 2000.400 Seiten, ca. $100 \mathrm{Abb}$. gebunden, ca. $€ 99$ ISBN 3-7985-1208-6
Anamnese, Haut-, In-vitround Provokationstests. Die einzelnen Tests sind sehr detailliert beschrieben mit zahlreichen praktischen Hinweisen zur Durchführung und Interpretation. Auch spezialisierte Verfahren kommen zur Darstellung, wie zelluläre Funktionstests, PhotoPatch- und Photo-Provokationstests, physikalische Tests bei Urtikaria und Stichprovokation. Zusätzlich werden die Qualitätssicherung von Allergen-Extrakten und der direkte Allergen-Nachweis behandelt.

Im zweiten Teil wird die klinische Diagnostik einzelner allergischer Krankheitsbilder oder assoziierter bzw. abzugrenzender Reaktionen dargestellt, wie z.B. anstrengungsinduzierte Urtikaria und Anaphylaxie oder „Multiple Chemical Sensitivity“. Außerdem werden auf das Atopie-Screening sowie die spezifische Immuntherapie eingegangen. Die Darstellungen in diesem Teil sind so umfassend (häufig auch kurze Abrisse zur Epidemiologie, Pathophysiolgie und Therapieprinzipien), dass das Buch an ein Lehrbuch der Gesamt-Allergologie grenzt. Allerdings ist die Diagnostik in ihren verschiedenen Facetten auch in diesem Teil betont und wird in jedem Krankheitskapitel ausführlich dargelegt, einschließlich der Diskussion von Konsequenzen der jeweiligen diagnostischen Erkenntnisse.

Zusammenfassend stellt das Werk eine hochaktuelle, vollständige und außerordentlich kompetente, fachüber- greifende Darstellung der allergologischen Diagnostik dar. Im ersten Teil können die einzelnen Testverfahren systematisch nachgelesen werden, im zweiten Teil das Vorgehen beim jeweiligen Krankheitsbild. Dies macht das Buch auch zu einem gut strukturierten Nachschlagewerk in der Praxis.

Prof. Dr. J. Saloga, Mainz

\section{Asthma aktuell}

D as Werk schildert ausführlich Epidemiologie, Ursachen und Pathophysiologie des Asthma bronchiale. Neben einem Abriss zur Diagnostik liegt sein Schwerpunkt auf die Therapie. Die Autoren erläutern die Pharmakologie der Wirkstoffe, einschließlich der neueren Leukotrien-Antagonisten. Das ChurgStrauss-Syndrom, als MedikamentenNebenwirkung, findet auf immerhin 20 Seiten Platz. Die unterschiedlichen Therapien von Asthma im Erwachsenensowie im Kindes- und Jugendalter werden unter Berücksichtigung der aktuellen Empfehlungen erläutert. Die $\mathrm{Be}-$ handlung des Aspirin-induzierten Asthmas sowie des Anstrengungsasthmas erhalten eigene Kapitel - ebenso die noch nicht verfügbaren Anti-IgE-Antikörper. Schließlich werden die Inhalationstherapie und die Möglichkeiten einer Compliance-Verbesserung dargestellt.

Bei kritischer Durchsicht scheint der erhebliche Umfang der Abschnitte zu Leukotrien-Antagonisten der Bedeutung der Substanzen nicht angemessen zu sein. Auch ist die Bewertung der therapeutischen Maßnahmen nicht immer ganz ausgewogen. Unerfreulich ist die nicht sachgerechte Darstellung der spezifischen Immuntherapie. Dennoch gehört diese 2. Auflage zu den besten aktuellen Informationsquellen über das Krankheitsbild Asthma bronchiale.

Prof. G. Schultze-Werninghaus, Bochum

Kroegel C, Hrsg. Asthma bronchiale.

Pathogenetische Grundlagen, Diagnostik,

Therapie. Stuttgart: Thieme, 2002. 341 Seiten, 48 Abb., $€ 24,95$. ISBN 3-13-104732-1 